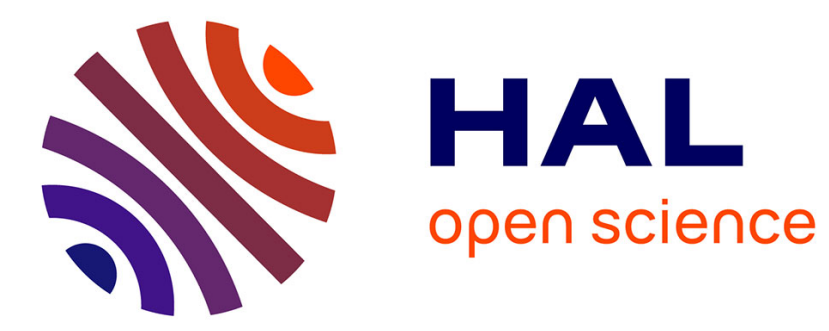

\title{
Subpicosecond excitonic optical nonlinearities in quantum wells
}

M. Joffre, D. Hulin, A. Migus, A. Mysyrowicz, A. Antonetti

\section{To cite this version:}

M. Joffre, D. Hulin, A. Migus, A. Mysyrowicz, A. Antonetti. Subpicosecond excitonic optical nonlinearities in quantum wells. Revue de Physique Appliquée, 1987, 22 (12), pp.1705-1709. 10.1051/rphysap:0198700220120170500 . jpa-00245730

\section{HAL Id: jpa-00245730 https://hal.science/jpa-00245730}

Submitted on 1 Jan 1987

HAL is a multi-disciplinary open access archive for the deposit and dissemination of scientific research documents, whether they are published or not. The documents may come from teaching and research institutions in France or abroad, or from public or private research centers.
L'archive ouverte pluridisciplinaire HAL, est destinée au dépôt et à la diffusion de documents scientifiques de niveau recherche, publiés ou non, émanant des établissements d'enseignement et de recherche français ou étrangers, des laboratoires publics ou privés. 
Classification

Physics Abstracts

$71.35-42.50 \mathrm{H}-42.65 \mathrm{R}-73.40 \mathrm{~L}$

\title{
Subpicosecond excitonic optical nonlinearities in quantum wells
}

\author{
M. Joffre, D. Hulin, A. Migus, A. Mysyrowicz and A. Antonetti \\ Laboratoire d'Optique Appliquée, ENS Techniques Avancées, Ecole Polytechnique, 91120 Palaiseau, France
}

(Reçu le 25 juin 1987, accepté le 24 août 1987)

\begin{abstract}
Résumé. - Les résonances excitoniques dans les semiconducteurs sont particulièrement sensibles à l'action d'un champ lumineux. Nous avons comparé les effets d'une excitation optique, selon qu'elle est résonnante ou non résonnante, c'est-à-dire qu'elle crée des excitons réels ou virtuels. On observe dans les structures à puits quantiques multiples un décalage vers le bleu des raies excitoniques accompagné par une diminution de la force d'oscillateur de la résonance dans les deux cas suivants : présence d'une population d'excitons dans des puits très étroits (systèmes bi-dimensionnels), ou présence d'un champ optique intense non résonnant (création virtuelle d'excitons) quelle que soit la largeur du puits. Cette dernière configuration, dite effet Stark optique, a été étudiée en fonction de l'intensité de l'excitation lumineuse, permettant de déterminer en particulier la relation qu'il y a entre la saturation du décalage de la raie et l'écrantage de l'absorption excitonique.
\end{abstract}

\begin{abstract}
Excitonic resonances in semiconductors are very sensitive to applied optical fields. We present here a comparison between the effects of resonant and non resonant optical excitation, creating respectively real or virtual excitons. A blue shift of the exciton line and a reduction of the oscillator strength are simultaneously observed in multiple-quantum-well-structures for the two following cases : presence of a population of real excitons in very thin wells (two-dimensional systems), or presence of an intense offresonance non absorbed optical field (virtual excitons) independently of the well thickness. The latter configuration (optical Stark effect) has been studied as a function of pump intensity, determining in particular how the saturation of the blue shift is related to the exciton bleaching.
\end{abstract}

Multiple Quantum Well Structures (MQWS) are currently attracting great interest both from a fundamental point of view as well as for their potential application as devices in electronics and in optics. Very large optical nonlinearities in MQWS are associated with the presence of excitonic resonances even at room temperature. Recent studies of the subpicosecond nonlinearities have shown two main mechanisms in the GaAs-GaAlAs MQWS. The first one occurs when the semiconductor is excited above the band gap, leading to the creation of carriers or excitons ; the second one occurs when the excitation is below the band gap yielding the so-called optical Stark effect. We have investigated the dynamics of these optical nonlinearities especially when induced by the optical Stark effect on a subpicosecond timescale.

We have performed time-resolved experiments using the classical pump and probe scheme. The primary pulse of $100 \mathrm{fs}$ duration at $620 \mathrm{~nm}$ is provided by a passively mode-locked ring dye laser. Its energy is then raised to an energy level of $0.5 \mathrm{~mJ}$ through four stages of amplification pumped by a frequency-doubled Nd-YAG laser at $10 \mathrm{~Hz}$ [1]. The beam is then split into two parts, one of the beams travelling through a variable optical path. Two white-light continua of similar duration as the initial pulse are generated in each path by focusing the beam into different water cells. The pump wavelength is selected by an interferential filter. The pump pulse is then further amplified to an energy of a few $\mu \mathrm{J}$ through a two-pass amplification stage pumped by part of the same Nd-YAG laser. The excitation beam is focused on a $0.1 \mathrm{~mm}^{2}$ area leading to an available energy density of $0.5 \mathrm{~mJ} / \mathrm{cm}^{2}$. The pump duration is mainly determined by the limit imposed by the spectral width (typically $3 \mathrm{~nm}$ ) of the interferential filter and therefore ranges usually around $300 \mathrm{fs}$. The probe pulse uses the other strongly-attenuated broad-band continuum; its $100 \mathrm{fs}$ duration determines the time resolution of the experiment. The polarization vector of the probe 
can be set either parallel or perpendicular to that of the pump. The spectrum of the probe pulse transmitted through the pump-irradiated sample is recorded at different delays between pump and probe pulses, providing a subpicosecond time-resolved picture of the absorption of the sample. The MQW structures of different well and barrier size have been grown by molecular beam epitaxy and are held at $T=15 \mathrm{~K}$.

Large changes in the excitonic absorption are observed under optical excitation above or at the exciton absorption resonances. In case of non-resonant pumping (band to band transition), the absorption of a pump photon corresponds to the creation of an electron and a hole. When the intensity of the pump light is increased, a reduction of the excitonic absorption is observed whose magnitude is related to the density and the temperature of the excited free carriers in the system (Fig. 1). No concommitant change in the energy of the excitonic resonances can be detected. This behaviour is independent of the 2or 3-dimensional nature of the sample, being observed in MQWS as well as in bulk GaAs. It is now well argued that this constancy of the exciton energy comes from an almost exact compensation between two many-body effects : a band gap renormalization which induces a rigid lowering of all the energy levels, and simultaneously a reduction of the exciton binding energy which, at $T=0 \mathrm{~K}$, compensates exactly the first effect [2].

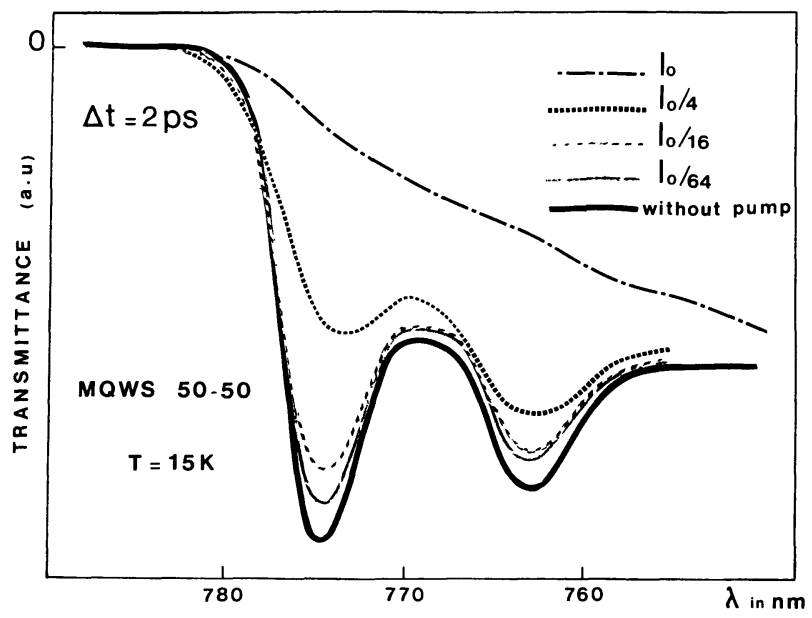

Fig. 1. - Transmittance spectra of a GaAs-AlGaAs MQWS at $15 \mathrm{~K}$ in presence of free carriers for different pump intensities. The spectra are recorded 2 ps after the creation of the carriers, before the recombination into excitons. The wells and the barriers are both of $52 \AA$ width.

The situation is quite different when a large number of excitons are present in the MQWS [3]. Experimentally this population can be either directly created by resonant pumping (direct initial injection of excitons by tuning the pump wavelength inside the lowest, heavy-hole exciton absorption line) or, at low temperature, by pumping in the band to band absorption and waiting for the pairing at later times of initially free charged carriers. We first consider the case of independent quasi two-dimensional systems which are quite well represented by MQWS of small well width (less than $100 \AA$ ). In presence of a large density of excitons, we observe a high energy shift of the excitonic resonances (Fig. 2). The mag-

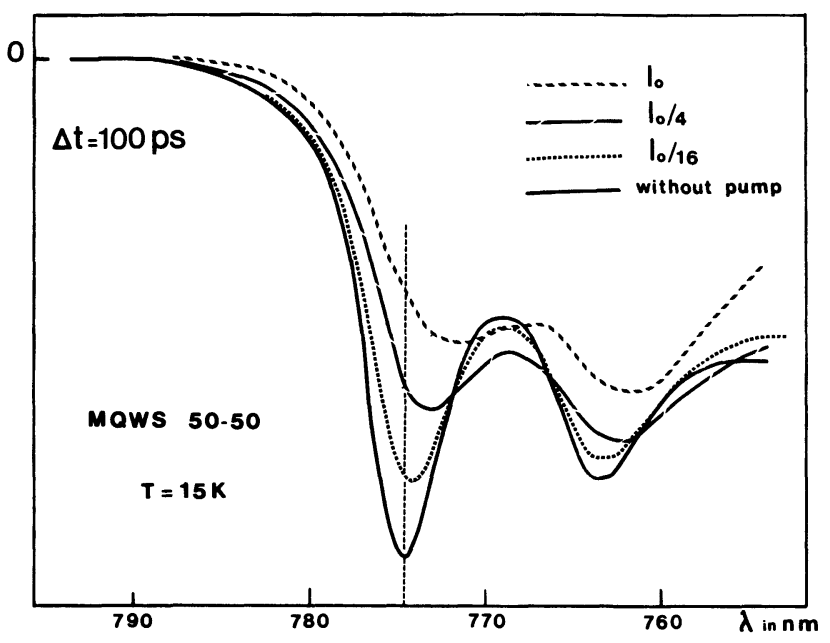

Fig. 2. - Transmittance spectra of a GaAs-AlGaAs MQWS at $15 \mathrm{~K}$ in presence of excitons for different pump intensities. The excitons are obtained from the recombination of free carriers (100 ps after their creation).

nitude of this blue shift depends linearly upon the injected particle density at moderate pump intensities. The other noticeable feature is the simultaneous occurrence of a reduction of the oscillator strength. We have experimentally shown [4] that these two effects are related through the relationship :

$$
\Delta E / E_{\mathrm{x}}=c \Delta f / f
$$

where $\Delta E$ is the magnitude of the blue shift, $E_{\mathrm{x}}$ is the exciton binding energy and $\Delta f / f$ is the relative decrease of the excitonic oscillator strength. The proportionality coefficient $c$ is 1 for the heavyhole exciton and 0.5 for the light-hole exciton. The results are different in samples of larger well size. For the same experimental conditions, the reduction of the excitonic resonance absorption is much more pronounced and the blue shift is hardly observable. We have obtained a value $c=0.15$ in the case of $100 \AA$ thick wells and even smaller values for larger wells or bulk GaAs. This is consistent with the nonobservation of exciton shift in 3-dimensional materials.

The physical origin of the blue shift lies in the short-range hard-core repulsion of the excitons [5]. 
To get a full picture one has to take into account the short range exchange effects and the phase space filling which are both resulting from the Pauli exclusion principle, and the long-range direct Coulomb correlations. For an electron or a hole, Pauli exclusion prevents the creation of a particle in a state which is already occupied. Excitons are bosons but their wave functions are built as a linear combination of fermion states. The creation of an exciton corresponds then to a finite occupation probability in fermion space $[5,6]$. As it happens with the usual absorption saturation phenomenon, the phase space filling effect leads to a reduction of the oscillator strength proportional to the exciton density. Much the same can be said for the exchange process. Together with the bleaching of the excitonic resonance, the short range hard-core repulsion of the excitons produces also a blue shift proportional to the exciton density. This implies a linear relationship between excitonic resonance shift and saturation. We have also to consider the long-range Coulomb correlations. It is well known [7] that in case of reduced dimensionality the screening of the exciton by charged particles is reduced. The polarization of the exciton, which is a neutral entity, by an other exciton (long-range van der Waals attraction) is even more inefficient. Consequently the overall behaviour for excitons in perfect 2-D systems can be described by neglecting the long range direct Coulomb correlations. The situation is different when the structure has wider wells and can no more be considered as a two-dimensional system. The increasing influence of the screening leads to a small reduction of the blue shift but also to a more pronounced reduction of the oscillator strength, implying the quasi non-observation of the exciton shift in 3-D samples.

In the case of the optical Stark effect [8, 9], the situation is very different but presents also strong analogies with the previous case. The pump wavelength is now tuned inside the spectral transparency region of the MQWS. When the temperature of the sample is low, no absorption tail due to the simultaneous absorption of a photon and a phonon can take place so that no real particle should be created in this experimental configuration (except by two-photon absorption process). However the optical spectra is perturbed by the presence of an intense pump pulse as can be seen in figure 3. Absorption spectra of a MQWS of $100 \AA$ thick wells, recorded at a time delay corresponding to the maximum of the pump pulse, are displayed for different pump intensities. The pump wavelength $827 \mathrm{~nm}$ corresponds to a detuning of $46 \mathrm{meV}$ with respect to the lowest absorption line, i.e. the $\mathrm{h}-\mathrm{h}$ exciton $(802.5 \mathrm{~nm})$. The most striking feature lies in the blue shift of the excitonic resonances. A shift of $8 \mathrm{meV}$ can be observed for the $h-h$ exciton, indicating that the

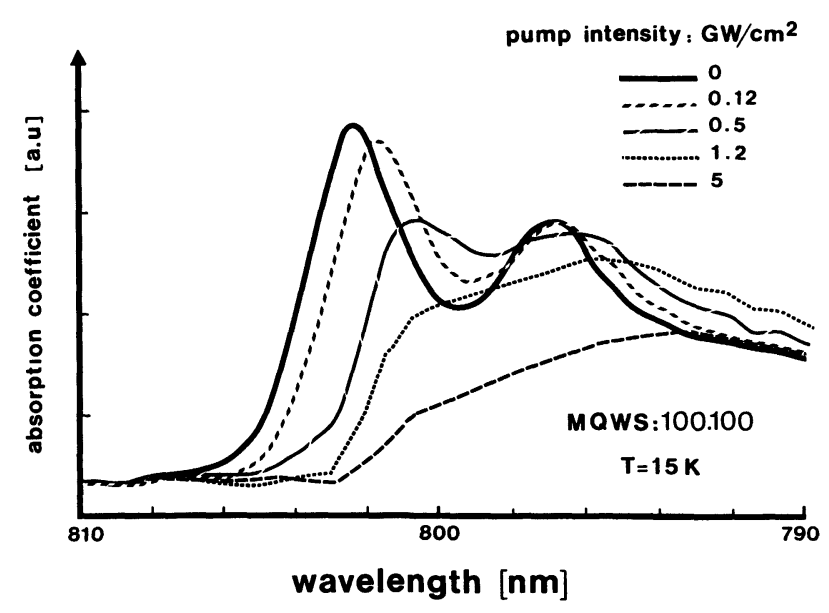

Fig. 3. - Time-resolved absorption spectra of a MQWS at $15 \mathrm{~K}$ during the presence of an intense optical pulse at $827 \mathrm{~nm}$ for different pump intensities. The wells and the barriers are both of $100 \AA$ width.

effect can be significantly large. The light hole exciton is also affected but in a smaller way; we have measured a factor 3 to 4 in the magnitude of the energy shift for the $1-h$ in comparison with the $h$ $\mathrm{h}$ exciton. The other noticeable feature in figure 3 is again a decrease of the exciton oscillator strength simultaneous with the line shift. For the highest intensity, an almost complete disappearence of the $\mathrm{h}$-h line occurs, making it difficult to measure the shift in this extreme case. The observed broadening is not very large and may partly be due to some fluctuations of the pump intensity although a small window $( \pm 20 \%)$ was applied to select pump pulses. All these effects are smaller for the $1-h$ exciton. After the end of the pump pulse the excitonic lines are again at the same position as before the excitation. Figure 4 shows the differential transmission in func-

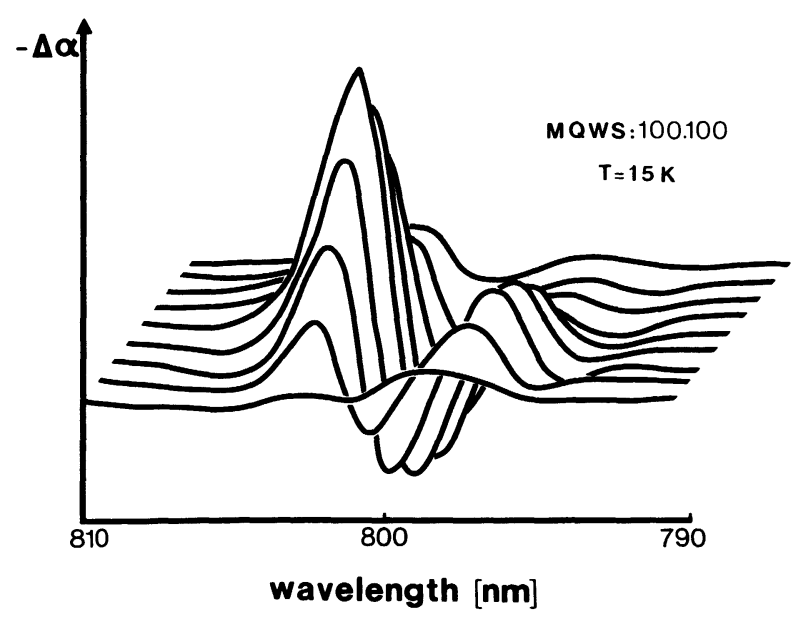

Fig. 4. - Differential absorption coefficient at different time delays (100 fs apart) corresponding to the non resonant excitation (optical Stark effect). The $\mathrm{h}-\mathrm{h}$ exciton is at $802.5 \mathrm{~nm}$ and the $1-\mathrm{h}$ at $797 \mathrm{~nm}$. 
tion of time of the MQWS irradiated with a pump pulse at $827 \mathrm{~nm}$ of $0.5 \mathrm{GW} / \mathrm{cm}^{2}$ intensity. The shift of the resonance shows up as a differential behaviour superimposed on a positive contribution due to the bleaching. The signal follows the cross-correlation of the pump and the probe within our experimental accuracy. A small remaining bleaching can be seen at longer times, indicating the presence in the system of a small number of photoexcited carriers.

We have interpreted this instantaneous blue shift in the frame of the simple model of the dressed atom. It is indeed well known in atomic physics that a two-level system non resonantly irradiated by a strong electromagnetic wave exhibits a shift of its resonance. At the lowest order this shift is due to the virtual creation and recombination of excited states (here excitons) which are both stimulated by the pump field. This process can also be understood as the virtual absorption and reemission of a pump photon and therefore implies two interactions with the pump field, which means that we have to develop the theory to second order. Following such a second order perturbation theory, the energy shift is proportional to the square of the electric field, to the oscillator strength and inversely proportional to the detuning between the pump frequency and the exciton energy. The exact atomic physics calculation yields the following expression :

$$
\Delta E / \hbar=\sqrt{\Delta \omega^{2}+\Omega^{2}}-\Delta \omega
$$

where $\Delta \omega$ is the detuning and $\Omega$ the Rabi frequency given by :

$$
\Omega=2 e E_{\mathrm{p}} \sqrt{f / 2 m \hbar \omega_{\mathrm{x}}}
$$

where $f$ is the oscillator strength, $E_{\mathrm{p}}$ the pump electric field and $\hbar \omega_{x}$ the exciton energy. Therefore at the lowest order the shift is :

$$
\Delta E=a f I_{\mathrm{p}} / \Delta \omega
$$

where $a$ is a constant and $I_{\mathrm{p}}$ the pump intensity. We may now question this oversimplified model since it is based on the assumption that the exciton is a two level system. A sophisticated many-body theory developed by Schmitt-Rink et al. [10] led however that to lowest order the shift is given by :

$$
\Delta E=2\left|\mu E_{\mathrm{p}}\right|^{2}\left|\phi_{1 \mathrm{~s}}(r=0)\right|^{2} / \hbar \Delta \omega N_{\mathrm{s}}
$$

where $\mu$ is the interband dipole matrix element, $\phi_{1 \mathrm{~s}}$ the $1 \mathrm{~s}$ exciton wave function and $N_{\mathrm{s}}$ the saturation density due to excitonic phase filling with $N_{\mathrm{s}}=7 / 8 \pi a_{0}^{2}$ for a two dimensional exciton gas of Bohr radius $a_{0}$. This implies that excitons can be considered as $N_{\mathrm{s}}$ two-level systems [11], justifying therefore our very simple argument.
The shift is associated with a strong reduction of the oscillator strength. Figure 5 plots the relative exciton energy shift with respect to the exciton binding energy versus the relative decrease of the oscillator strength variation. It follows a linear law with a slope of 1 , very similar to what is observed in the resonant case for purely $2-\mathrm{D}$ systems.

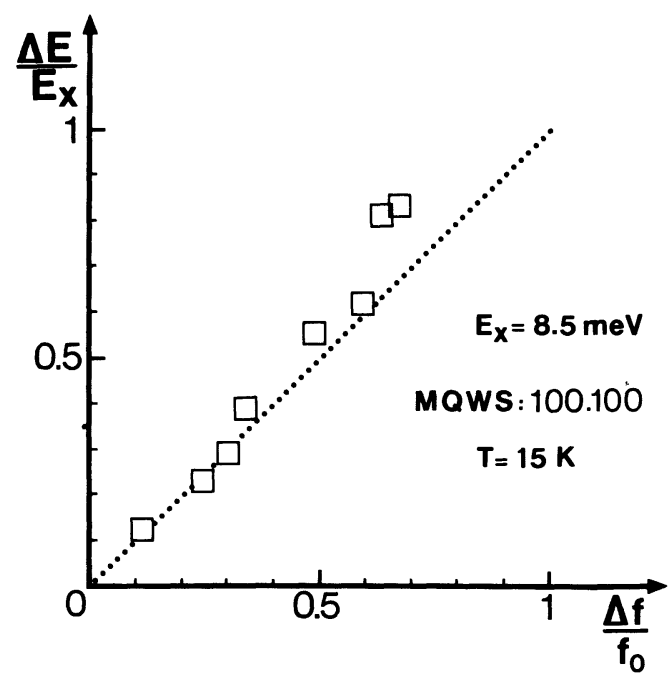

Fig. 5. - Relative h-h exciton energy shift in function of the relative decrease of the oscillator strength.

The figure 6 displays the dependence of the shift on the pump intensity. In the low power regime, the shift is linear with the pump intensity, as it is expected in a lowest order perturbation theory. But by using very high pump intensities (up to $10 \mathrm{GW} / \mathrm{cm}^{2}$ ), we were able to enter into the regime where the shift begins to saturate. The linear law (4) is the development of expression (2) valid when the Rabi frequency is much lower than the pump

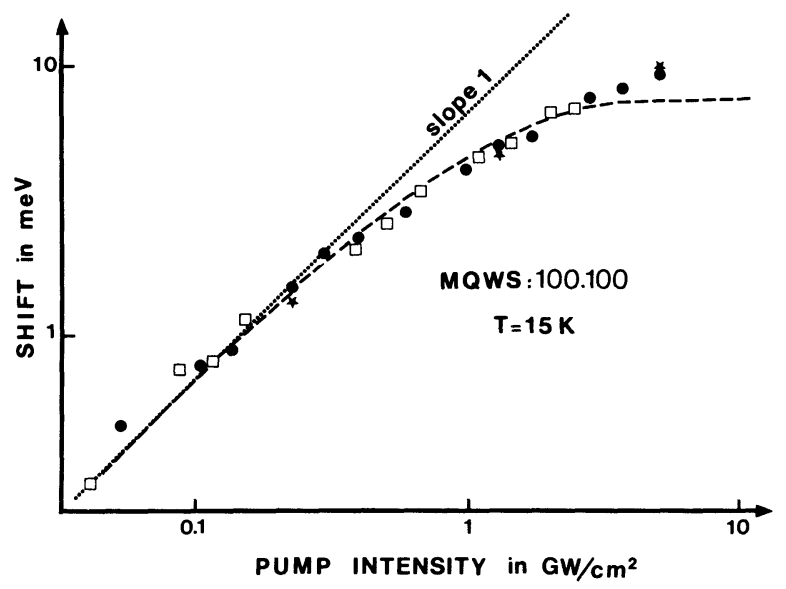

Fig. 6. - Variation of the h-h exciton energy in function of the pump intensity on a log-log scale. The dotted line corresponds to the linear law, the dashed line to expression (8) in the text. 
detuning. One would therefore expect an inflexion of the curve when $\Omega$ is of the order of magnitude of $\Delta \omega$, but in that case the shift itself would be of the order of the detuning. This is very far from our experimental observations so that we conclude that the square root law does not explain the non-linear behaviour. We suggest that the saturation of the shift is actually related to the strong reduction of the oscillator strength. Let us consider an exciton perturbed by a strong electromagnetic field. Its energy is shifted by $\Delta E$ and we experimentally know its oscillator strength dependence :

$$
f=f_{0}-\Delta f=f_{0}\left(1-\Delta E / E_{\mathrm{x}}\right) .
$$

If we now make the drastic assumption that the expression (4) is valid even for an exciton perturbed by a strong electromagnetic field, we obtain the following differential equation between the shift and the pump intensity

$$
\mathrm{d} \Delta E=a / \Delta \omega f_{0}\left(1-\Delta E / E_{\mathrm{x}}\right) \mathrm{d} I_{\mathrm{p}} .
$$

Integrating this equation, we obtain the relation between the shift and the pump intensity:

$$
\Delta E=E_{\mathrm{x}}\left[1-\exp \left(a f_{0} I_{\mathrm{p}} / \Delta \omega E_{\mathrm{x}}\right)\right] .
$$

We have reported the corresponding curve in figure 6 . The only adjustable parameter is $a$, and as can be seen the fit with the experimental points is very good.

The transition from the ground state to the excited state of the semiconductor with the destruction of two pump photons and the creation of one photon of energy $2 \omega_{p}-\omega_{x}$ could be stimulated by a test beam, leading to an optical gain. The two-level theory predicts that this gain is at maximum a few percent of the probe field absorption. This indeed has been observed in a pure two-level atomic system such as sodium atoms [12]. More work are presently being done in that field concerning excitons.

In summary, we have used femtosecond techniques to study the dynamics of the optical nonlinearities of the exciton in GaAs MQWS. A high energy shift of the excitonic resonance occurs in two different experimental conditions, following that a large population of real or virtual excitons are created. In the former case the blue shift of the excitonic lines is a pure bidimensional effect, while in the latter it appears as well in bulk or 2-D systems. This instantaneous nonlinear optical mechanism has already been exploited to operate a GaAs/AlGaAs optical gate with subpicosecond switch on and off times [13].

\section{Acknowledgements.}

We are very grateful to Pr. Morkoç and W. T. Masselink for the elaboration of the high quality multiple quantum well structures. We wish to thank S. Schmitt-Rink for making us his paper available prior publication. We would like to thank D.R.E.T. (grant $\mathrm{n}^{\circ} 86089$ ) and N.A.T.O. (travel grant 86/0749).
[1] Migus, A., Antonetti, A., Etchepare, J., Hulin, D. and Orszag, A., J. Opt. Soc. Am. 2 (1985) 584.

[2] Zimmermann, R., Killmann, K., Kraeft, W. A., Kremp, D. and Ropke, G., Phys. Status Solidi b 90 (1978) 175.

[3] Peyghambarian, N., Gibbs, H. M., Jewell, J. L., Antonetti, A., Migus, A., Hulin D. and Mysyrowicz, A., Phys. Rev. Lett. 53 (1984) 2433.

[4] Hulin, D., Mysyrowicz, A., Antonetti, A., Migus, A., Masselink, W. T., Morkoç, H., Gibbs, H. M. and Peyghambarian, N., Phys. Rev. B 33 (1986) 4389.

[5] Schmitt-Rink, S., Chemla, D. S. and Miller, D. A. B., Phys. Rev. B 32 (1985) 6601.

[6] Nozières, P. and Comte, C., J. Physique 43 (1982) 1083.

[7] Ando, T., Fowler, A. B. and Stern, F. Rev. Mod. Phys. 54 (1982) 437.
[8] Mysyrowicz, A., Hulin, D., Antonetti, A., Migus, A., Masselink, W. T. and Morkoç, H., Phys. Rev. Lett. 56 (1986) 2748.

[9] Von Lehmen, A., Chemla, D. S., Zucker, J. E. and Heritage, J. P., Opt. Lett. 11 (1986) 609.

[10] Schmitt-Rink, S. and Chemla, D. S., Phys. Rev. Lett. 57 (1986) 2752.

[11] Chemla, D. S., Schmitt-Rink, S. and Miller, D. A. B., to be published in Nonlinear Optical Properties of Semiconductors, Ed H. Haug (Academic New York) 1987.

[12] Wu, F. Y., Ezekiel, S., Ducloy, M. and Mollow, B. R., Phys. Rev. Lett. 38 (1977) 1077.

[13] Hulin, D., Mysyrowicz, A., Antonetti, A., Migus, A., Masselink, W. T., Morkoç, H., Gibbs, H. M. and Peyghambarian, N., Appl. Phys. Lett. 49 (1986) 749. 